\title{
Experimental Investigation on the Interfacial Debonding between FRP Sheet and Concrete under Medium Strain Rate
}

\author{
Zihua Zhang $\mathbb{D}^{1,2}$ Yunyi Xiao, ${ }^{1}$ Ping Zhuge $\mathbb{D}^{1},{ }^{1}$ and Xiaocun Zhang $^{1}$ \\ ${ }^{1}$ School of Civil and Environmental Engineering, Ningbo University, 315211, China \\ ${ }^{2}$ Key Laboratory of Impact and Safety Engineering, Ministry of Education, Ningbo University, Ningbo, 315211, China
}

Correspondence should be addressed to Ping Zhuge; zhugeping@nbu.edu.cn

Received 30 July 2019; Revised 23 October 2019; Accepted 5 November 2019; Published 18 December 2019

Academic Editor: Maria Laura Di Lorenzo

Copyright (C) 2019 Zihua Zhang et al. This is an open access article distributed under the Creative Commons Attribution License, which permits unrestricted use, distribution, and reproduction in any medium, provided the original work is properly cited.

\begin{abstract}
Fiber-reinforced polymer (FRP) composites have been widely used to strengthen the existing reinforced concrete (RC) structures to against static and dynamic loads. During the past decades, the interfacial bond behavior between FRP and the concrete substrate under static load has been systematically investigated by experimental and numerical approaches. In contrast, the interfacial bond performance under dynamic loads, e.g., impact and explosive loading, is still far away from well known, especially taking the strain rate effect into account. In this contribution, the single-lap shear test is conducted to sixty specimens at the medium strain rate between $1.0 E-4 / \mathrm{s}$ and $5.0 E-3 / \mathrm{s}$. The effects of various system parameters, including the strain rate, concrete strength, type of FRP and adhesive, on the interfacial fracture energy, peak shear stress, FRP strain distribution, interfacial shear stress, and effective bond length, are thoroughly investigated. It has been revealed that the strain rate and concrete strength can significantly affect the interfacial fracture energy and peak shear stress. The specimen with CFRP sheet possesses higher interfacial shear stress but lower fracture energy than that with BFRP sheet. The adhesive with lower elastic modulus is helpful to improve interfacial energy dissipation under dynamic load. The effective bond length decreases with concrete strength and strain rate, mainly between $75 \mathrm{~mm}$ and $90 \mathrm{~mm}$, which is significantly shorter than that under static load. Inspired from the Kulkarni and Shah model, a new model is proposed to evaluate the interfacial fracture energy and peak shear stress with respect to the strain rate, and the estimated values agree well with the experiments.
\end{abstract}

\section{Introduction}

Concrete structures are frequently suffering from different types of damage in practical engineering, such as overloading, earthquake, erosion, and fire. Such inevitable phenomenon leads to a direct consequence of a reduction on the bearing capacity of the structure. In the past decades, different types of reinforcement technique, such as the externally bonded steel plate method [1], the prestress strengthening method [2], the externally bonded fiber-reinforced polymer (FRP) method [3], and the hybrid method [4], have been innovatively developed to rehabilitate or improve the performance of the damaged concrete members, so the initial designated service requirements can be met. Among these innovatively developed approaches, the externally bonded
FRP method possesses several attractive advantages including the negligible added weight, excellent durability, and convenient construction. FRP sheets or strips are directly pasted to the surface of the concrete substrate by epoxy resin adhesive, and then the reinforced structure becomes a typical composite consisting of concrete, FRP, and the interface between FRP and concrete. Despite of the advantages of the FRP that have been offered, the issue of limited strengthening efficiency, which is caused by the mercurial interfacial debonding effects, is still obstructing the full implementation in real-life engineering applications. Numerous experiments and numerical simulations have revealed that the interfacial bond-slip relationship under static loading condition is affected by a series of factors, including concrete strength, FRP type, stiffness of adhesive, and interfacial bond length 
and width [5-11]. Reviews on the debonding strengthen models can be found in [12], and different types of anchorage devices used to achieve higher levels of fiber utilization prior to premature debonding failure are reviewed in [13].

It is worth noting that the FRP-reinforced structures may suffer dynamic loads, e.g., seismic load, explosion, impact, and crash, in the subsequent usage [14-16]. In these cases, owning to the strain rate effect, the interfacial bond-slip relationship between the FRP and concrete is much different from the situations under static and quasistatic loads. Table 1 [17] lists the strain rates under different loading cases. To date, there are only a few literatures covering the effect of strain rate on the interfacial bond-slip relationship. Through double-lap shear tests with strain rate ranging from $1.0 E-5 / \mathrm{s}$ to $1.0 E-2 / \mathrm{s}$, Shi et al. [18] found that the concrete strength is an important factor affecting the strain rate effect of the FRP-concrete interface. Shen et al. [19] presented an experimental investigation on the dynamic effective bond length between the basalt fiber-reinforced polymer (BFRP) sheets and concrete under various strain rates. It was found that dynamic effective bond length of the BFRP-concrete interface is affected by both the BFRP stiffness and concrete strength, and it decreases in the form of a logarithmic function as the strain rate increases. The maximum bond stress, ultimate load, and bond-slip relationship are also sensitive to the strain rate [20].

On the other hand, numerical approaches, e.g., FEM, are employed to simulate the interfacial debonding behavior between FRP and concrete under dynamic loads, but it is still a challenge to achieve robust results efficiently owning to the nonlinear behavior of interfacial debonding between FRP and concrete. To name a few, Caggiano et al. [21] proposed a modified Duvaut-Lions zero-thickness interface model to simulate the strain rate effect in FRP sheets glued to concrete substrate. Chen et al. [22] treated the debonding failure as a dynamic problem and solved using a time integration method to overcome the convergence problem. Lin and Zhang [23] performed a nonlinear analysis using LS-DYNA to investigate the effect of strain rate effect on the blast resistance of FRP-strengthened reinforced concrete panels.

In order to reveal the interfacial debonding mechanism between FRP sheet and concrete substrate concerning the strain rate, single-lap shear tests are utilized to sixty specimens under different strain rates between $1.0 E-4 / \mathrm{s}$ and $5.0 E-3 / \mathrm{s}$, and the effect of different factors, including strain rate, FRP type, concrete strength, and adhesive type, on the interfacial fracture energy, peak shear stress, strain, and bond-slip curves, is comprehensively investigated by parametric analysis. The Kulkarni and Shah model [24], which was initiated for pure concrete, is extended to estimate the interfacial mechanical performance between the FRP and concrete under medium strain rate.

The rest of the paper is organized as follows. The details of the single-lap shear test are illustrated in Section 2. In Section 3, the experimental results are investigated, and the effects of concrete strength, strain rate, FRP, and adhesive type on the interfacial bearing capacity and features are thoroughly discussed. A brand new, yet effective, model for evaluating the interfacial fracture energy and peak shear stress considering the strain rate effect is proposed and verified in Section 4 . Section 5 concludes the paper.

\section{Experimental Program}

2.1. Material Properties. The grade of 32.5R Portland cement produced by Anhui Conch Cement Co., Ltd. is used to make sixty specimens with the concrete grade of C20, C30, and $\mathrm{C} 40$, using ISO standard sand as the fine aggregate and gravel with $5 \mathrm{~mm}$ to $20 \mathrm{~mm}$ diameter as the coarse aggregate. The components of concrete are listed in Table 2. The compressive strength and tensile strength of the concrete are tested by GB/T 50081-2002. Two types of FRP (CFRP and BFRP) and two types of epoxy resin adhesive (both produced by HITECH Nanjing and here denoted as adhesive A and adhesive Q) are used. The material properties of FRP and adhesive are tested by GB/T 3354 and GB/T 2568, respectively, and listed in Table 3.

2.2. Specimen Dimensions and Loading Rate. Single-lap shear specimens are used to test the interfacial debonding process, as shown in Figure 1. The FRP sheet is pasted in a premarked area $(50 \mathrm{~mm} \times 200 \mathrm{~mm})$ on the top of concrete block with roller compression to exclude bubbles and improve the interfacial debonding performance. Note that the bond length is over the effective bond length estimated from the previous model [20]. An unbonded length of $50 \mathrm{~mm}$ was reserved to eliminate the effect of concrete edge [25]. Nine strain gauges are arranged along the centerline of the FRP layer. Considering that the stress gradient is relatively steep near the free end, the strain gauge spacing near the free end is less than that away from the free end. The single-lap shear specimen is fixed by the anchoring device as shown in Figure 2, and the strain gauges are connected to the dynamic strain gauge by data acquisition lines. The steel clamp, FRP sheet, and fixed base are arranged in the same plane to eliminate the eccentric effect.

The loading rates are set as $200 \mathrm{~N} / \mathrm{s}, 2,000 \mathrm{~N} / \mathrm{s}, 6,000 \mathrm{~N} / \mathrm{s}$, and $10,000 \mathrm{~N} / \mathrm{s}$. Assuming that the force is applied uniformly along the loading end of FRP sheet, the corresponding strain rate at this position can be obtained as $1.0 E-4 / \mathrm{s}, 1.0 E-3 / \mathrm{s}$, $3.0 E-3 / \mathrm{s}$, and $5.0 E-3 / \mathrm{s}$, mainly to simulate the situation under seismic load as shown in Table 1.

2.3. Test Program. Under the static loading condition, it has been well revealed that the interfacial bonding properties between FRP and concrete are affected by many factors, such as concrete strength, FRP properties, adhesive properties, and bond length and width. For dynamic cases, it has been found that the strain rate, FRP type, concrete strength, and adhesive type are major factors affecting the interfacial debonding performance [18]; thus, we adopt these four factors as the system parameters, as shown in Table 4. Here, the letter "I" denotes the quasistatic load, and "II," "III," and "IV" denote dynamic load with different load speeds (i.e., different strain rates), respectively. For instance, a specimen named C20AI1-3 uses CFRP, C20 concrete, and adhesive A under quasistatic load. In each group, three specimens are tested, and the average value is used for subsequent analysis. 
TABLE 1: Magnitude of strain rates expected for different loading cases [17].

\begin{tabular}{lcccc}
\hline Load type & Creep & Static & Earthquake & Hard impact \\
Strain rate $\left(\mathrm{s}^{-1}\right)$ & $10^{-8} \sim 10^{-6}$ & $10^{-6} \sim 10^{-4}$ & $10^{-3} \sim 10^{-2}$ & $10^{0} \sim 10^{2}$ \\
\hline
\end{tabular}

TABLE 2: Components and mix proportion of the concrete.

\begin{tabular}{lcccccc}
\hline \multirow{2}{*}{ Concrete strength grade } & \multicolumn{4}{c}{ Mix portion of components } & \multirow{2}{*}{ Compressive strength $f_{c u}(\mathrm{MPa})$} & \multirow{2}{*}{ Tensile strength $f_{p}(\mathrm{MPa})$} \\
& Cement & Gravel & Sand & Water & 21.6 & 1.55 \\
C20 & 1 & 0.75 & 2.72 & 4.35 & 31.0 & 2.21 \\
C30 & 1 & 0.51 & 1.66 & 3.21 & 45.9 & 2.45 \\
C40 & 1 & 0.41 & 1.21 & 2.64 & & \\
\hline
\end{tabular}

TABLE 3: Material properties of FRP and adhesive.

\begin{tabular}{lcccc}
\hline $\begin{array}{l}\text { Material } \\
\text { type }\end{array}$ & $\begin{array}{c}\text { Tensile } \\
\text { strength } f_{p} \\
(\mathrm{MPa})\end{array}$ & $\begin{array}{c}\text { Elastic } \\
\text { modulus } E_{f} \\
(\mathrm{GPa})\end{array}$ & $\begin{array}{c}\text { Elongation } \\
\delta(\%)\end{array}$ & $\begin{array}{c}\text { Nominal } \\
\text { thickness } t_{f} \\
(\mathrm{~mm})\end{array}$ \\
\hline CFRP & 3570 & 240 & 1.7 & 0.111 \\
BFRP & 2045 & 80.5 & 2.7 & 0.156 \\
$\begin{array}{l}\text { Adhesive } \\
\text { A }\end{array}$ & 55.5 & 3.2 & 2.2 & $/$ \\
$\begin{array}{l}\text { Adhesive } \\
\text { Q }\end{array}$ & 40.8 & 2.4 & 3.1 & $/$ \\
\hline
\end{tabular}
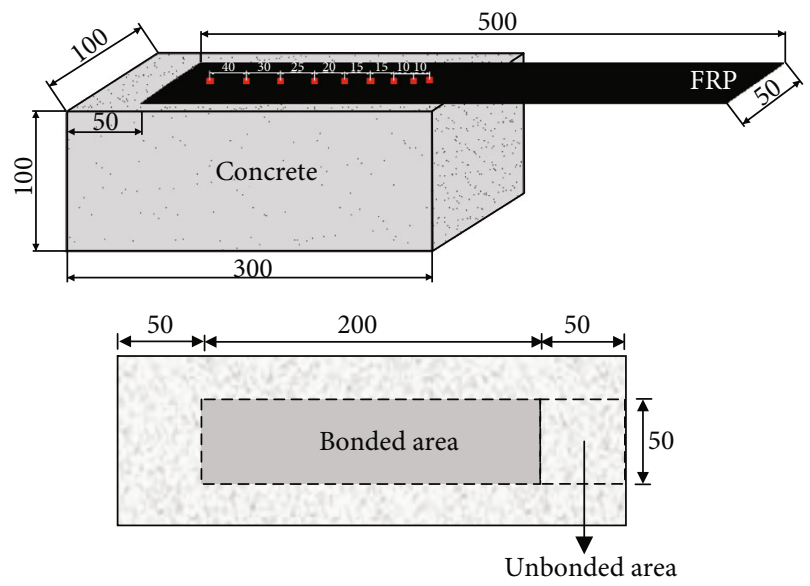

FIGURE 1: Specimen dimensions of the single-lap shear test (unit: $\mathrm{mm}$ ).

For each type of specimen, three samples are tested and the average values of these three samples are used. The BFRP is double layered to achieve similar stiffness to CFRP.

\section{Experimental Results and Discussion}

3.1. Interfacial Debonding Process and Failure Modes. At the initial stage of loading, a small relative slip between the FRP sheet and the concrete substrate was observed as a slight noise can be heard. By increasing the load, the FRP sheet with a thin mortar layer near the FRP-concrete interface was gradually peeled off from the concrete substrate. When the ulti- mate damage load was reached, the FRP sheet and concrete substrate were instantly separated with no obvious premonition. It can be observed that the destructive area of the concrete substrate was slightly larger than bonding area, similar as the phenomenon observed under static loading condition. According to the experimental results, the failure modes can be classified into three types (see Figure 3): (i) interfacial cohesion debonding between the FRP sheet and concrete substrate, which occurs within $2 \mathrm{~mm}$ to $5 \mathrm{~mm}$ below the concrete surface; (ii) internal shear failure of adhesive layer; and (iii) rupture of the FRP sheet. Assuming that the quality of construction is ensured, the first mode of failure is acceptable and will be discussed in the subsequent section.

3.2. Interfacial Fracture Energy. Interfacial fracture energy $G_{f}$ is usually used to evaluate the bearing capacity of the interface, which equals to the envelop area of the bond-slip curve and can be denoted as follows [26]:

$$
G_{f}=\frac{P_{u}^{2}}{2 b_{f}^{2} E_{f} t_{f}},
$$

where $P_{u}$ is the ultimate shear load, and $b_{f}, t_{f}$, and $E_{f}$ are the width, nominal thickness, and elastic modulus of the FRP sheet, respectively.

The effects of strain rate and material properties on $G_{f}$ are depicted in Figure 4 with the experimental data and linear fitting curves. In Figure 4(a), it is observed that $G_{f}$ increases with the concrete strength and the specimens using lower-strength concrete have a more obvious strain rate effect. Considering the debonding failure occurs in a thin layer of concrete beneath the interface, the strain rate effect is mainly dependent on the concrete strength. Figure 4(b) reveals that the specimens with BFRP possess higher $G_{f}$ than those with CFRP, because BFRP has a lower stiffness and a better ductility to dissipate the fracture energy. Such a phenomenon was also observed in [18]. The linear fitting curves of CFRP and BFRP are parallel to each other, which indicates that the FRP type only changes the values of $G_{f}$, but the trends for different types of FRP are similar. In Figure 4(c), it is found that the specimens using adhesive A possess lower $G_{f}$, indicating that the adhesive with lower elastic modulus 

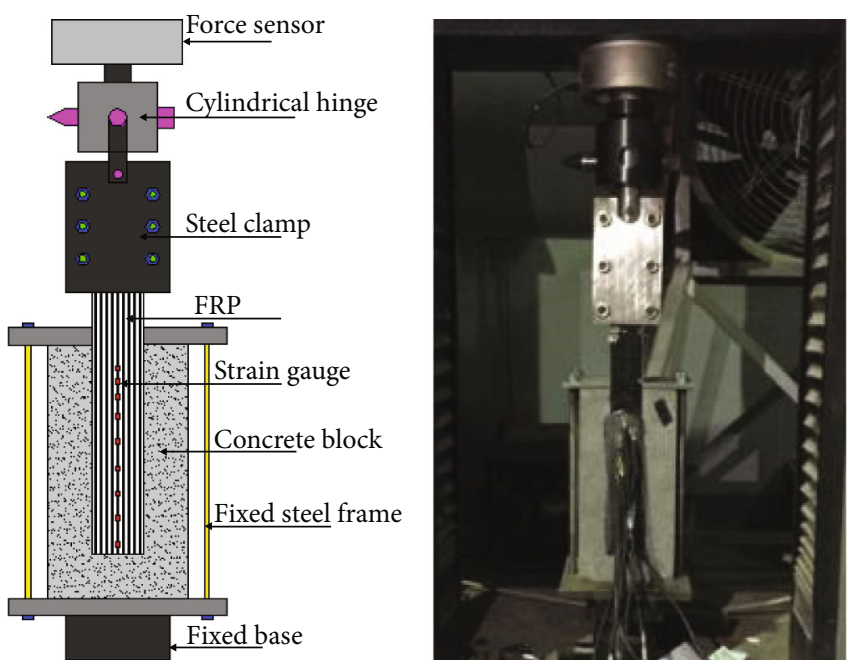

Figure 2: Loading device of single-lap shear test.

TABle 4: Test program and results.

\begin{tabular}{|c|c|c|c|c|c|}
\hline $\begin{array}{l}\text { Specimen } \\
\text { no. }\end{array}$ & $\begin{array}{l}\text { FRP } \\
\text { type }\end{array}$ & $\begin{array}{c}\text { Concrete } \\
\text { strength } \\
\text { grade }\end{array}$ & $\begin{array}{l}\text { Adhesive } \\
\text { type }\end{array}$ & $\begin{array}{c}\text { Strain } \\
\text { rate }\left(s^{-1}\right)\end{array}$ & $\begin{array}{c}\text { Ultimate } \\
\text { shear load } \\
P_{u}(\mathrm{kN})\end{array}$ \\
\hline C20AI & \multirow{4}{*}{ CFRP } & \multirow{4}{*}{$\mathrm{C} 20$} & \multirow{4}{*}{ A } & $1.0 E-4$ & 9.51 \\
\hline C20AII & & & & $1.0 E-3$ & 10.62 \\
\hline C20AIII & & & & $3.0 E-3$ & 11.48 \\
\hline C20AIV & & & & $5.0 E-3$ & 12.23 \\
\hline C30AI & \multirow{4}{*}{ CFRP } & \multirow{4}{*}{ C30 } & \multirow{4}{*}{ A } & $1.0 E-4$ & 10.02 \\
\hline C30AII & & & & $1.0 E-3$ & 11.12 \\
\hline C30AIII & & & & $3.0 E-3$ & 11.88 \\
\hline C30AIV & & & & $5.0 E-3$ & 12.19 \\
\hline C40AI & \multirow{4}{*}{ CFRP } & \multirow{4}{*}{ C40 } & \multirow{4}{*}{ A } & $1.0 E-4$ & 11.33 \\
\hline C40AII & & & & $1.0 E-3$ & 11.88 \\
\hline C40AIII & & & & $3.0 E-3$ & 12.12 \\
\hline C40AIV & & & & $5.0 E-3$ & 12.45 \\
\hline B30AI & \multirow{4}{*}{ BFRP } & \multirow{4}{*}{ C30 } & \multirow{4}{*}{ A } & $1.0 E-4$ & 10.23 \\
\hline B30AII & & & & $1.0 E-3$ & 11.20 \\
\hline B30AIII & & & & $3.0 E-3$ & 11.69 \\
\hline B30AIV & & & & $5.0 E-3$ & 12.11 \\
\hline C30QI & \multirow{4}{*}{ CFRP } & \multirow{4}{*}{ C30 } & \multirow{4}{*}{ Q } & $1.0 E-4$ & 10.12 \\
\hline C30QII & & & & $1.0 E-3$ & 10.98 \\
\hline C30QIII & & & & $3.0 E-3$ & 12.04 \\
\hline C30QIV & & & & $5.0 E-3$ & 12.59 \\
\hline
\end{tabular}

contributes to higher interfacial fracture energy. A possible reason is that more energy is dissipated by the viscoelastic adhesive layer.

3.3. FRP Strain Distribution. Assuming the FRP and adhesive are linear elastic, the average shear stress between the adja-

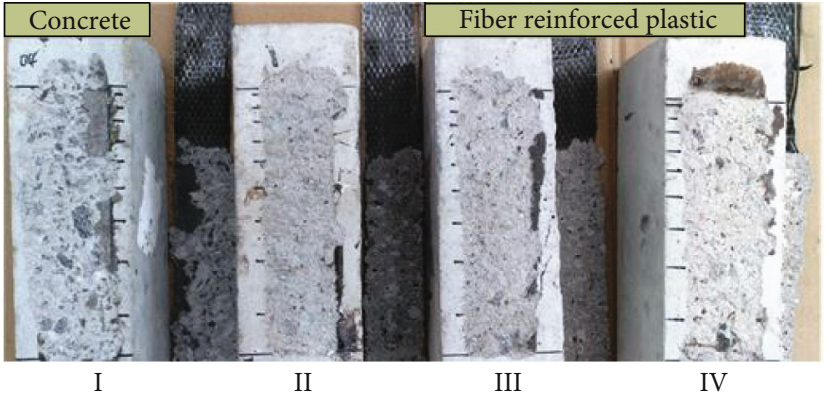

Figure 3: Interfacial debonding of single-lap shear test under different strain rates.

cent strain gauges can be calculated by [27]

$$
\tau_{f}=\frac{E_{f}\left(\varepsilon_{f 2}-\varepsilon_{f 1}\right) t_{f}}{\Delta x},
$$

where $\tau_{f}$ is the average shear stress, $\varepsilon_{f 1}$ and $\varepsilon_{f 2}$ are the strains of two adjacent strain gauges, respectively, and $\Delta x$ is the distance between two adjacent strain gauges.

Figure 5 shows the strain distribution of the FRP sheet and corresponding interfacial shear stress of typical specimens at different instants. Here, $t_{1}, t_{2}$, and $t_{3}$ denote the instant of the shear load reaching $0.2 P_{u}, 0.6 P_{u}$, and $1.0 P_{u}$, respectively. It can be observed that the stress wave propagates from loading end to the free end of the FRP sheet. At the beginning of loading $\left(t_{1}\right)$, the shear stress peak locates near the loading end, indicating that the debonding failure begins to occur at this section. As the load increases, the shear stress peak gradually transfers to the free end $\left(t_{2}\right.$ and $t_{3}$ ), and the debonding failure front also propagates towards the free end. After debonding, the interfacial shear stress suddenly decreases owning to unloading. From a trend perspective, for a specified instant and position, the strain peak and shear stress peak both increase with the strain rate. Comparing Figures 5(a)-5(d), it can be found that the strain 


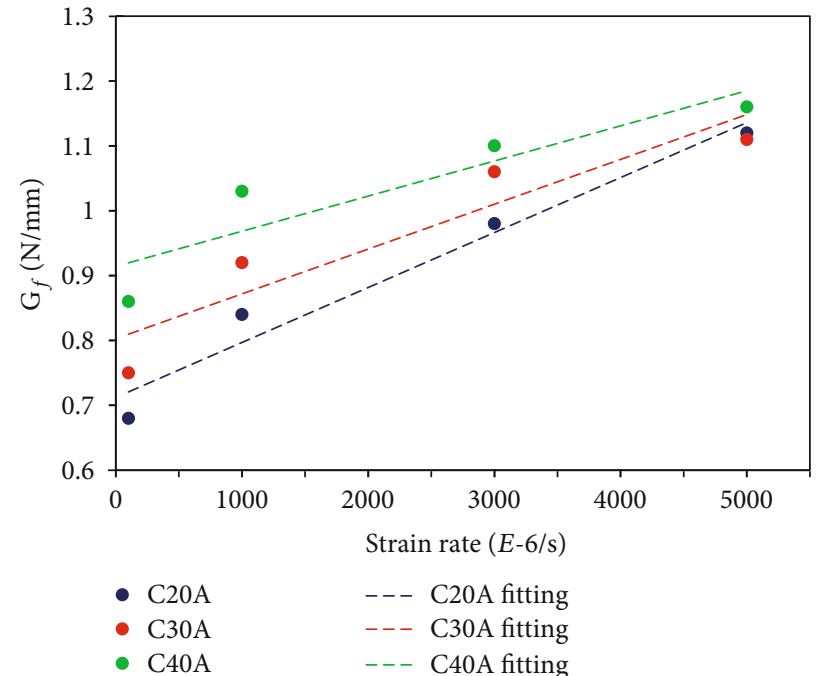

(a) Effect of concrete strength on $G_{f}$

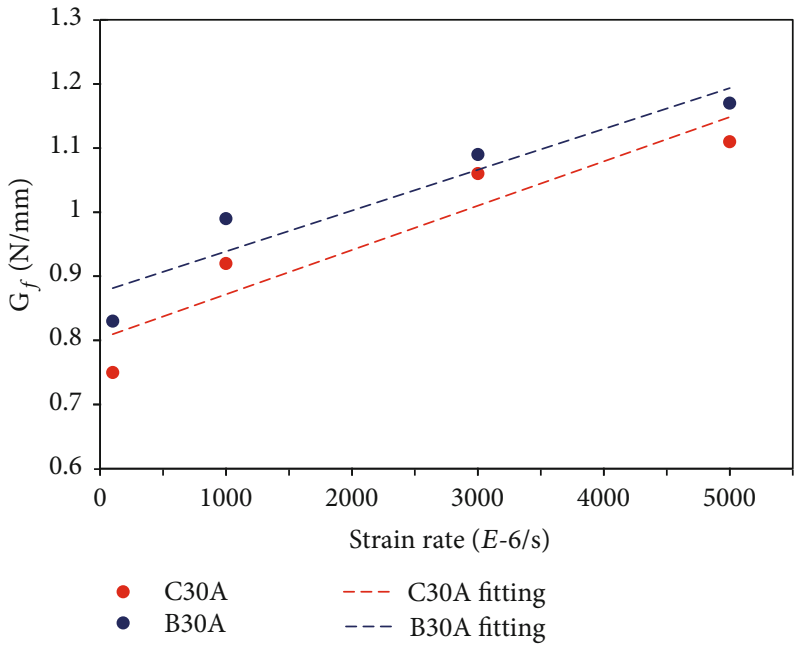

(b) Effect of FRP type on $G_{f}$

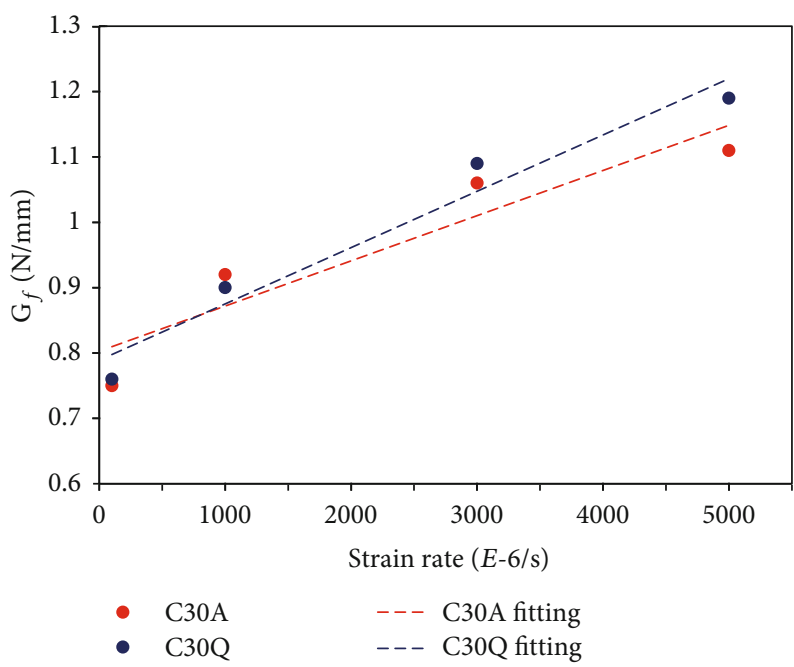

(c) Effect of adhesive type on $G_{f}$

FIGURE 4: Effect of material properties on $G_{f}$.

peaks of the specimens using $\mathrm{C} 40$ are $7.6 \%, 3.1 \%, 5.5 \%$, and $8.4 \%$ higher than those using $\mathrm{C} 30$ at four different strain rates. This indicates that the concrete with higher tensile strength can effectively increase the strain peak of the interfacial debonding.

As shown in Figures 5(a), 5(b), 5(e), and 5(f), the slope of strain distribution curves for the specimens using BFRP is slightly smaller than those using CFRP. Since the slope of the strain distribution curve represents the magnitude of the interfacial shear stress, it indicates that the interfacial shear stress of the specimens using CFRP is greater than those using BFRP during the debonding process, which is related to the stiffness of the FRP sheet. That is, the greater the stiffness of FRP sheet, the greater the interfacial shear stress. Figures 5(a), 5(b), 5(g), and 5(h) show the effect of adhesive type on the FRP strain and the interfacial shear stress distribution. The strain transfer area of the specimen using adhesive $\mathrm{Q}$ is about $40 \mathrm{~mm}$ shorter than that with adhe- sive A. That is, adhesive A transferred stress wave faster than adhesive Q. As seen from the material properties of the adhesive in Table 3, the tensile strength and elastic modulus of adhesive $\mathrm{A}$ are both higher than adhesive $\mathrm{Q}$, namely, adhesive A possesses a better resistance to longitudinal deformation than adhesive $\mathrm{Q}$, thus leading to a shorter time of interfacial debonding.

3.4. Interfacial Shear Stress. Equation (3) can be used to fit the FRP strain distribution by regression analysis [27].

$$
\varepsilon(x)=\frac{a / b}{1+e^{\left(x-x_{0}\right) / b}},
$$

where $a$ and $b$ are fitting parameters, $x$ is the distance from the loading end, and $x_{0}$ is the distance from the loading end to the position of the peak shear stress. 

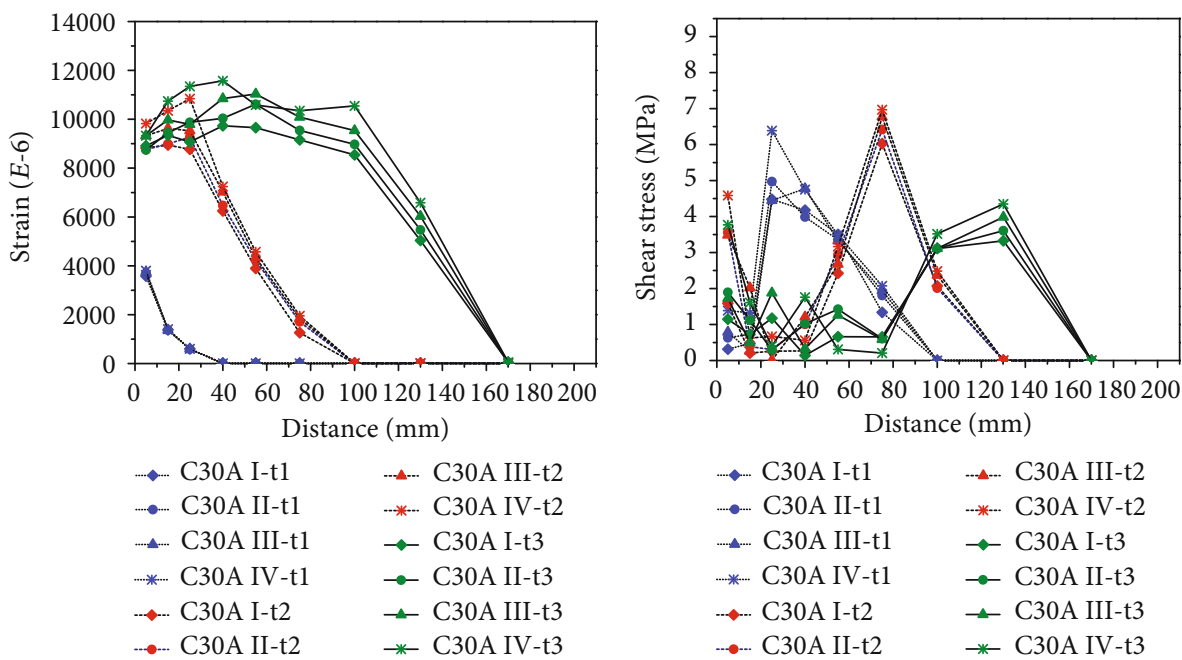

(a) Interfacial strain distribution for $\mathrm{C} 30 \mathrm{~A}$
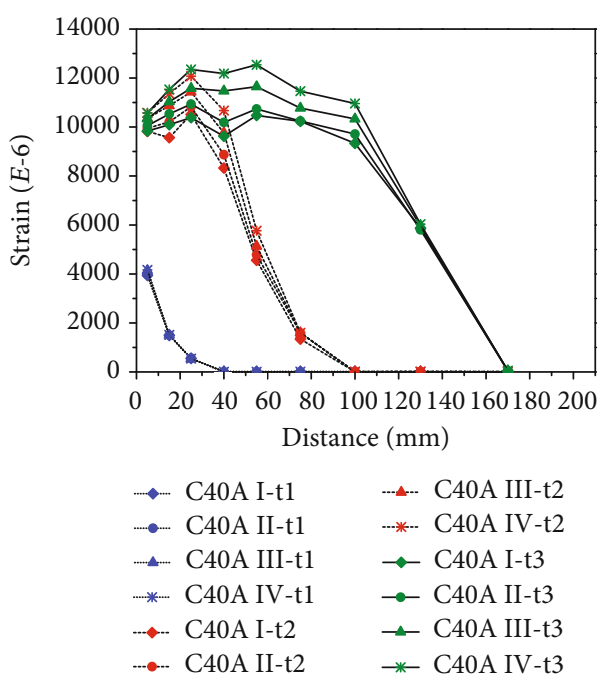

(c) Interfacial strain distribution for $\mathrm{C} 40 \mathrm{~A}$

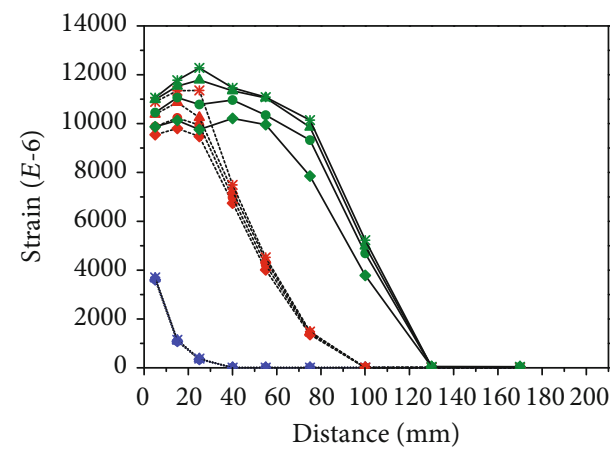

$$
\begin{aligned}
& \rightarrow \text { B30A I-t1 _..... B30A III-t2 } \\
& \text {-. B30A II-t1 …- B30A IV-t2 } \\
& \rightarrow \text { B30A III-t1 } \rightarrow \text { B30A I-t3 } \\
& \rightarrow \text { *... B30A IV-t1 } \rightarrow \text { B30A II-t3 } \\
& \leftrightarrow \text { B30A I-t2 } \_ \text {B30A III-t3 } \\
& \text {..... B B } 30 \mathrm{~A} \text { II-t2 } \rightarrow \text { - B30A IV-t3 }
\end{aligned}
$$

(e) Interfacial strain distribution for B30A
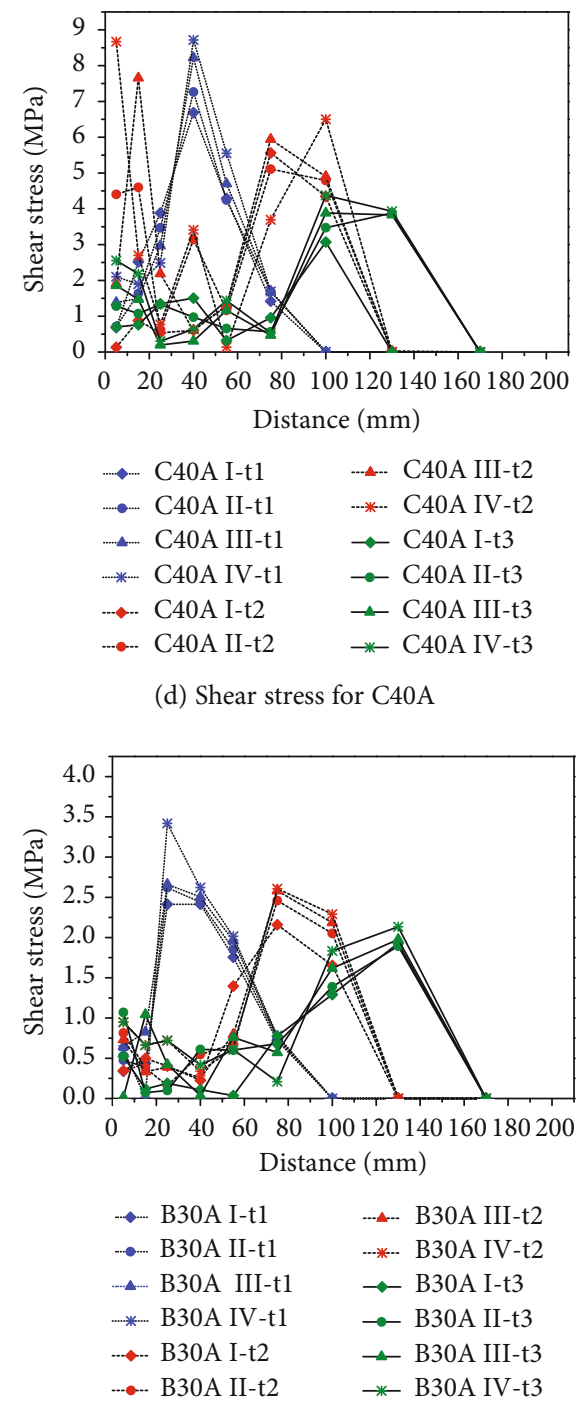

$$
\begin{aligned}
& \rightarrow \text { C30A I-t1 } \rightarrow \text { C30A III-t2 } \\
& \because \text { C30A II-t1 } \rightarrow \text { *-. C30A IV-t2 } \\
& \rightarrow \text { - C30A III-t1 } \rightarrow \text { C30A I-t3 } \\
& \text { * C30A IV-t1 } \rightarrow \text { C30A II-t3 } \\
& \rightarrow \text { C30A I-t2 } \rightarrow \text { C30A III-t3 } \\
& \text {.... C30A II-t2 } \quad \text { - } 2 \text { C } 30 \mathrm{~A} \text { IV-t3 }
\end{aligned}
$$

(b) Shear stress for C30A

$$
\begin{aligned}
& \rightarrow \text { C40A I-t1 } \quad \ldots \_ \text {C40A III-t2 } \\
& \rightarrow \text { C40A II-t1 } \rightarrow \text { *-. C40A IV-t2 } \\
& \rightarrow \text { C40A III-t1 } \rightarrow \text { C40A I-t3 } \\
& \text { *... C40A IV-t1 } \rightarrow \text { C40A II-t3 } \\
& \rightarrow \text { C40A I-t2 } \rightarrow \text { C40A III-t3 } \\
& \text {..- C40A II-t2 * C40A IV-t3 }
\end{aligned}
$$

(d) Shear stress for C40A

(f) Shear stress for B30A

FIgURe 5: Continued. 


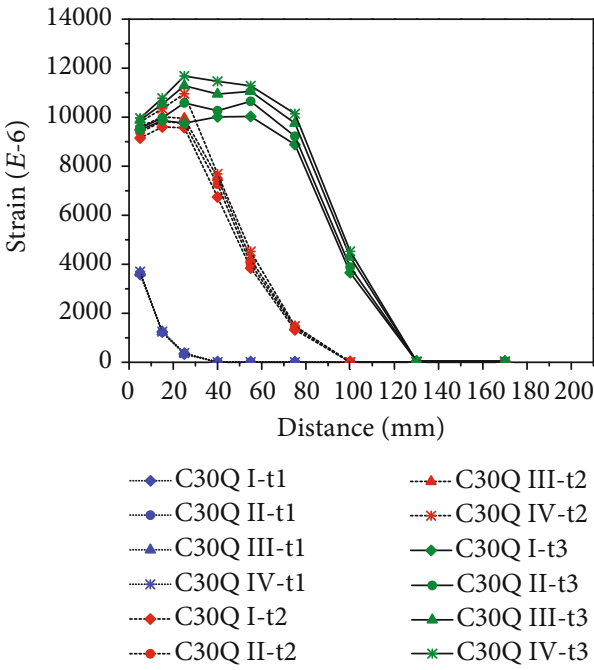

(g) Interfacial strain distribution for C30Q

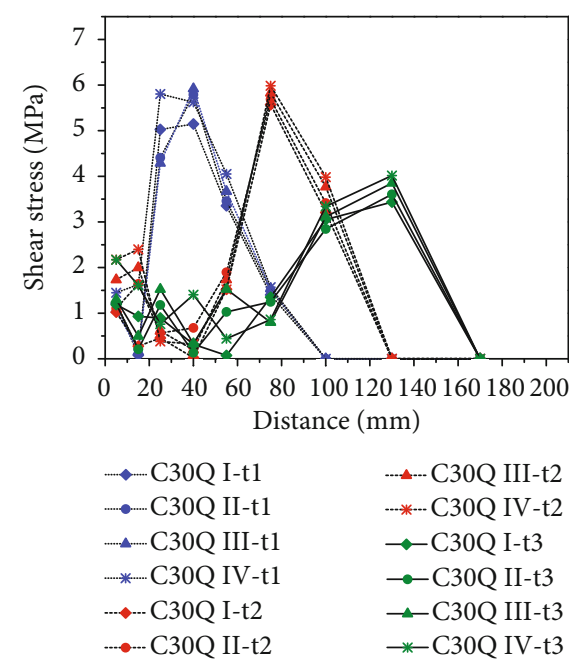

(h) Shear stress for C30Q

FIGURE 5: Interfacial strain distribution and shear stress for different specimens.

The first derivative of Equation (3) is as follows:

$$
\varepsilon(x)^{\prime}=-\frac{a}{b^{2}} \frac{e^{\left(x-x_{0}\right) / b}}{\left(1+e^{\left(x-x_{0}\right) / b}\right)^{2}} .
$$

The interfacial shear stress distribution can be expressed as follows:

$$
\tau(x)=\left|E_{f} t_{f} \mathcal{E}(x)^{\prime}\right|=\frac{a}{b^{2}} \frac{E_{f} t_{f} e^{\left(x-x_{0}\right) / b}}{\left(1+e^{\left(x-x_{0}\right) / b}\right)^{2}},
$$

where $E_{f}$ and $t_{f}$ are the elastic modulus and the thickness of the FRP, respectively.

Using Equation (5) to fit the interfacial shear stress distribution in Figure 5 by regression analysis, the fitting curves can be achieved. For concise, only typical curves related to Figure 5(a) are shown in Figure 6. Noting that, with the increase of loading time, the peak of the interfacial shear stress transfers from the loading end to the free end. Due to the homogeneous characteristics of concrete, the peak shear stresses are possessed at different positions along the interface; thus, the average value of the peak shear stresses is taken as the peak value of the specimen and depicted in Figure 6.

3.5. Effective Bond Length. It has been found that there exists an effective bond length (i.e., $L_{e}$ ) between FRP and concrete, indicating that the interfacial bearing capacity remains basically the same when the interfacial pasting length is over $L_{e}$. The estimation formula suggested by Dai et al. [26] is adopted to evaluate the strain rate effect on the effective bond length, which is

$$
L_{e}=2 b \ln \left(\frac{1+\eta}{1-\eta}\right)
$$

with $\eta=0.96$.
The $L_{e}$ of the specimens using C20A, C30A, and C40A with different strain rates are depicted in Figure 7. Under the quasistatic loading condition, $L_{e}$ equals to $89.5 \mathrm{~mm}$, $83 \mathrm{~mm}$, and $84.5 \mathrm{~mm}$ for $\mathrm{C} 20 \mathrm{~A}, \mathrm{C} 30 \mathrm{~A}$, and C40A, respectively, and the values agree with the observations of Chajes et al. [28] $(80 \mathrm{~mm})$ and Triantafillou et al. [29] $(90 \mathrm{~mm})$. It is also observed that $L_{e}$ gradually decreases with the strain rate. In the case of higher strain rate, the interfacial debonding occurs in a shorter instant and the stress wave has less time to propagation from the loading end to the free end; thus, a thicker and shorter layer of concrete is peeled off from the substrate to dissipate the peeling energy. Compared with the quasistatic condition, $L_{e}$ decreases by $10.1 \%, 8.4 \%$, and $5.8 \%$ for $\mathrm{C} 20 \mathrm{~A}, \mathrm{C} 30 \mathrm{~A}$, and $\mathrm{C} 40 \mathrm{~A}$ at the strain rate of $5.0 \mathrm{E}-3 / \mathrm{s}$, respectively, indicating that the specimens with lower concrete strength are more sensitive to the strain rate effect in terms of effective bond length.

3.6. Bond-Slip Relationship. The interfacial fracture energy $G_{f}$ and the peak shear stress $\tau_{\text {max }}$ represent the energy consumed per unit area of FRP-concrete interface and the magnitude of the ultimate shear stress, respectively. These two parameters not only reflect the magnitude of the interface ultimate failure load but also evaluate the interfacial adhesion, and always used to evaluate the bonding performance of the FRP-concrete interface. The slip of the interface can be obtained by the integral of strain (Equation (3))

$$
s(x)=\int \varepsilon(x) d x=a \ln \left(1+e^{\left(x_{0}-x\right) / b}\right)
$$

Using Equations (3), (5), and (6), the bond-slip relationship of the interface can be obtained as follows:

$$
\varepsilon(x)=\frac{a}{b}\left(1-e^{-s / a}\right),
$$




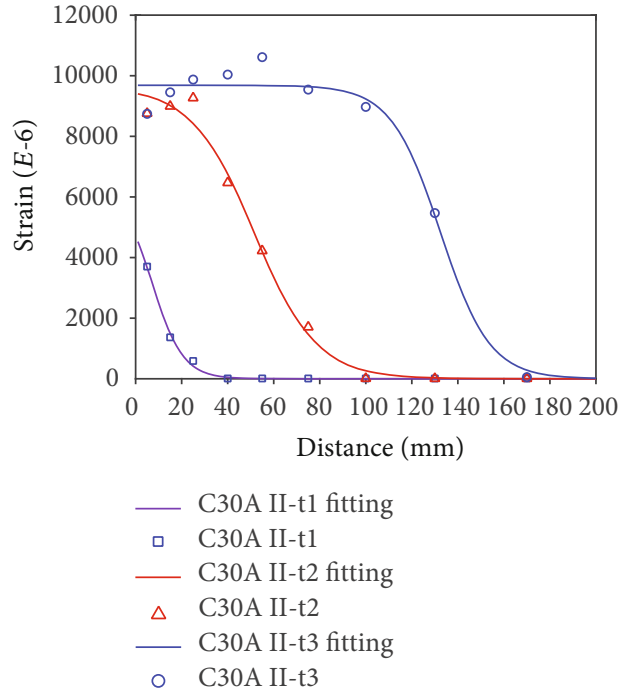

(a) Strain distribution on FRP sheet

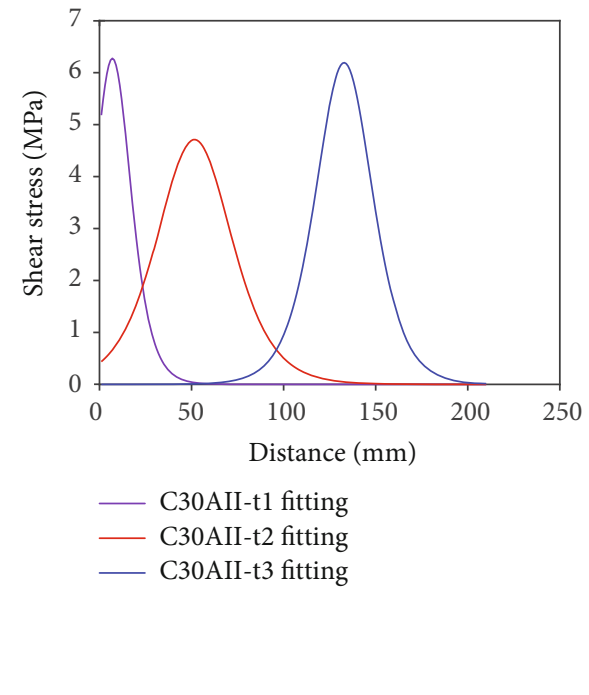

(b) Interfacial shear stress

FIgURE 6: Nonlinear fitting for the interfacial strain distribution and shear stress.

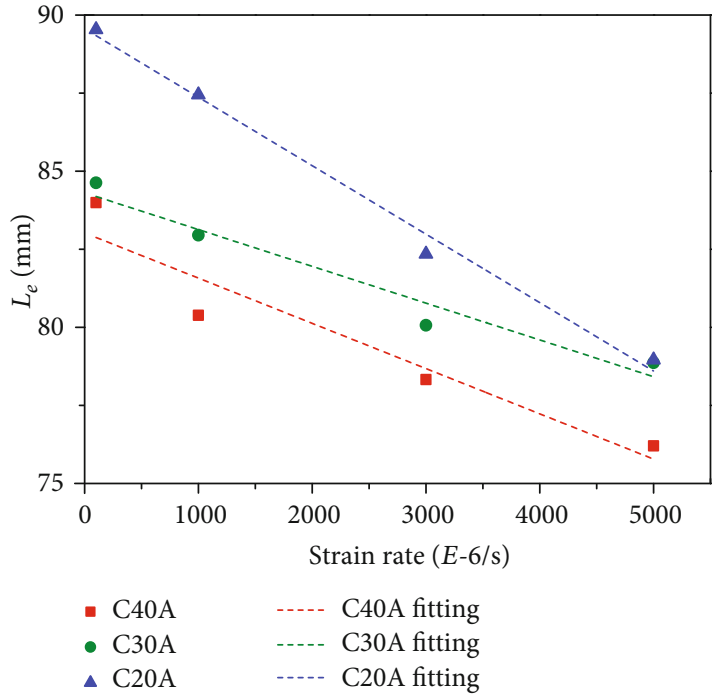

FIgURE 7: Strain rate effect on the effective bond length of CFRP.

$$
\tau(s)=E_{f} t_{f} \frac{a}{b^{2}} e^{-s / a}\left(1-e^{-s / a}\right)
$$

The bond-slip curves of all samples with different parameters are depicted in Figure 8. It can be seen that the shape of the curve is approximately in exponential shape, which can be divided into three sections: (i) linear raising section, in which the interfacial shear stress increases with the interfacial slip linearly, owning to that the bearing capacity of the interface is mainly from the adhesive bonding force; (ii) nonlinear raising section, during which the interfacial shear stress keeps raising with a slow-down rate until it reaches the peak. In this section, the bonding force between the concrete substrate and the adhesive resin begins to work; (iii) exponential softening sec- tion, in which the interfacial shear stress gradually decreases with the slip, because the interface begins to debonding from the loading end to the free end.

From Figures $8(\mathrm{a})-8(\mathrm{e})$, we can see that the shear stress peak increases with the strain rate. But, the location of the shear stress peak remains basically the same. By comparing Figures 8(a)-8(c), it can be found that the increase of the concrete strength is helpful to improve the interfacial bearing capacity. From Figures 8(b) and 8(d), it can be concluded that the specimens using BFRP possess better ductility than those with CFRP, leading to a higher stress peak and longer total slip and thus higher interfacial fracture energy. By comparing Figures 8(b) and 8(e), it is proved that the specimens using softer adhesive are able to consume more fracture energy during the interfacial debonding process, as observed in Figure 4(c).

3.7. Peak Shear Stress. Integrating Equation (9) with respect to $s$, the interface fracture energy is obtained as follows:

$$
G_{f}=\int \tau(s) d s=\frac{a^{2}}{2 b^{2}} E_{f} t_{f}
$$

$\tau(x)$ reaches the maximum value as $(d \tau(x)) / d x=0$ and can be expressed as follows:

$$
\tau_{\max }=\frac{1}{4} E_{f} t_{f} \frac{a}{b^{2}} .
$$

Figure 9 shows the relationship between $\tau_{\max }$ and the strain rate by means of linear fitting. It was found that $\tau_{\max }$ increases with the strain rate for the specimens with different materials. Figure 9(a) shows that under the conditions with the strain rates of $1.0 E-4 / \mathrm{s}, 1.0 E-3 / \mathrm{s}, 3.0 E-3 / \mathrm{s}$, and 5.0 $E-3 / \mathrm{s}, \tau_{\max }$ of the specimens using $\mathrm{C} 40$ are $12.0 \%, 9.6 \%$, 


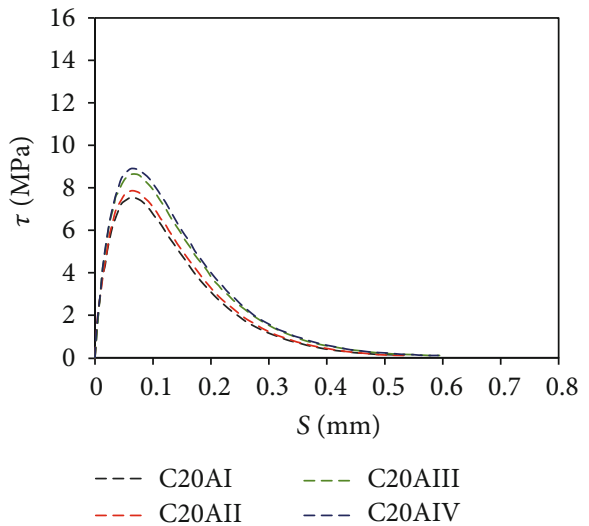

(a) $\mathrm{C} 20 \mathrm{~A}$

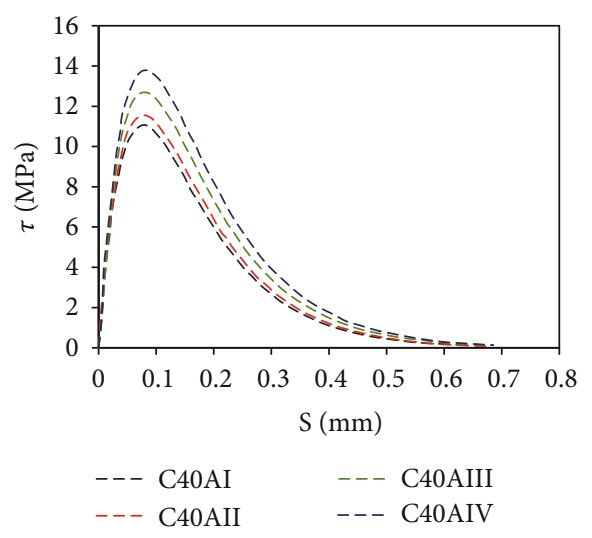

(c) $\mathrm{C} 40 \mathrm{~A}$

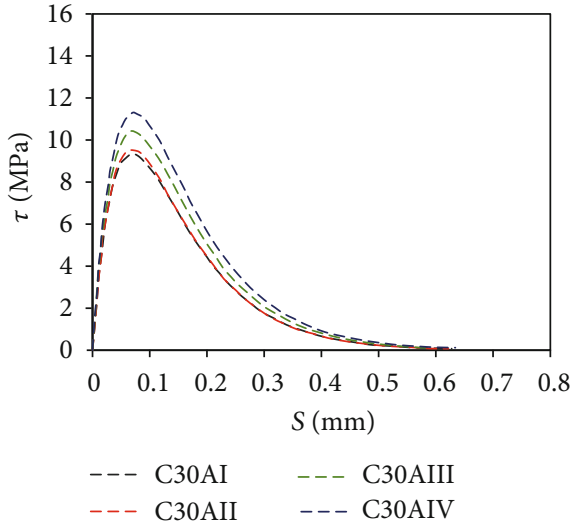

(b) $\mathrm{C} 30 \mathrm{~A}$

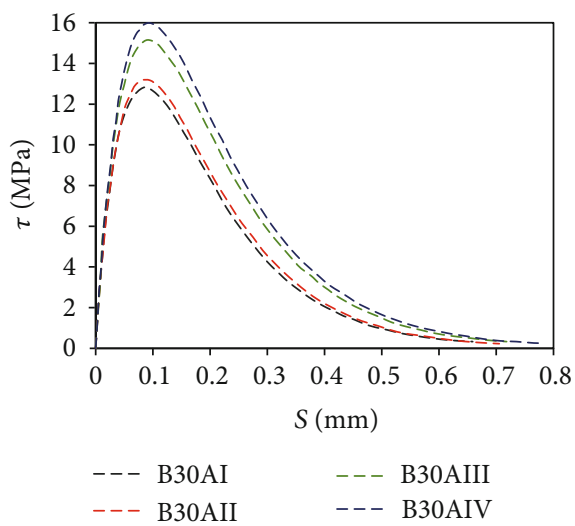

(d) $\mathrm{B} 30 \mathrm{~A}$

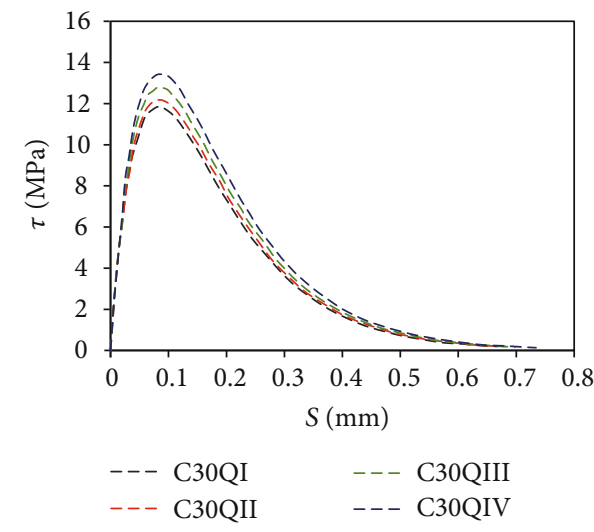

(e) C30Q

Figure 8: Bond-slip curves of the specimens.

4.2\%, and $2.3 \%$ higher than those using C30. The difference between the specimens using different types of concrete becomes smaller at high strain rate, because lower-strength concrete possesses more obvious strain rate. From Figure $9(\mathrm{~b})$, we can see that $\tau_{\max }$ of the specimens using BFRP are obviously lower than those using CFRP, which means the greater the stiffness of FRP, the higher peak shear stress. In addition, the fitting curves of CFRP and BFRP are almost parallel, which indicates that the FRP type only affects the value but not the trend of $\tau_{\max }$. Figures 9 (c) shows the influence of adhesive type on $\tau_{\text {max }}$, in which slight difference can be observed.

\section{Estimating Model for Interfacial Fracture Energy}

As mentioned above, the failure mode of the FRP-concrete interface under medium strain rate is cohesion failure in the concrete substrate, and the experimental results have revealed that the concrete strength is the major factor 


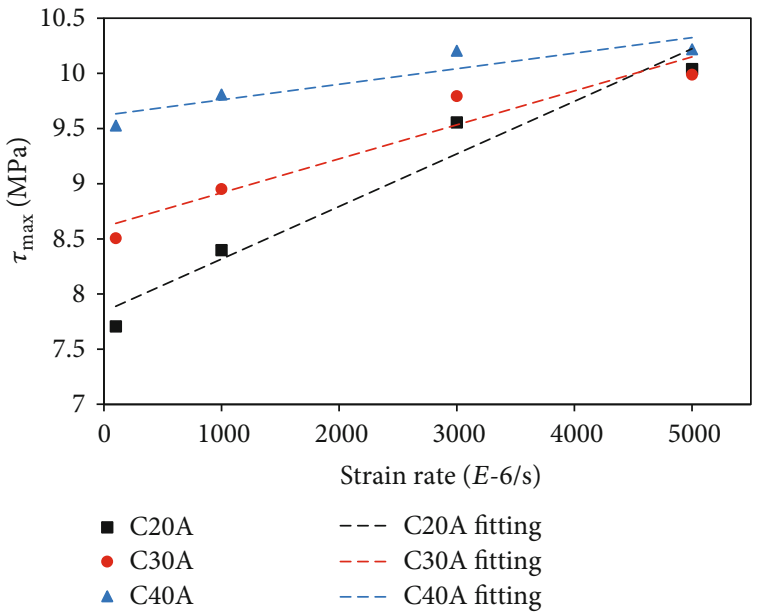

(a) Effect of concrete strength on $\tau_{\max }$

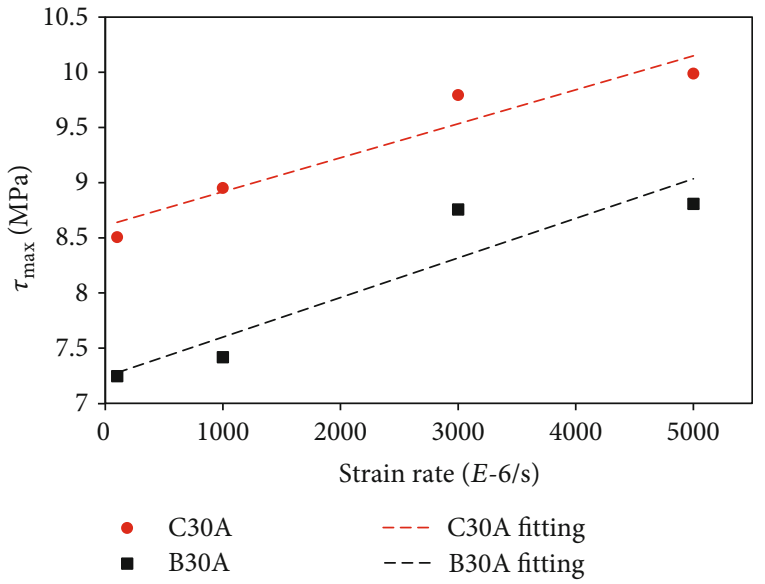

(b) Effect of FRP type on $\tau_{\max }$

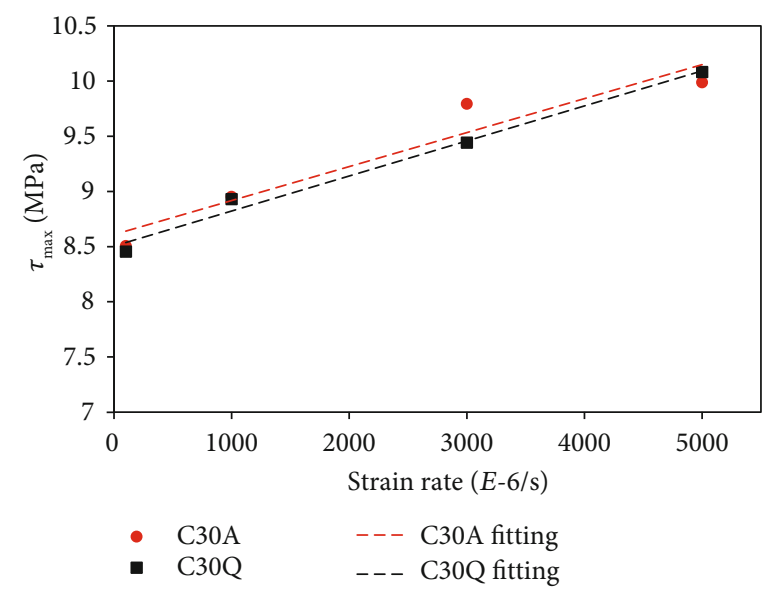

(c) Effect of adhesive type on $\tau_{\max }$

FIGURE 9: Effect of material properties on the strain effect of $G_{f}$ and $\tau_{\max }$.

affecting the strain rate effect of the interfacial debonding. Since concrete is a strain rate-sensitive material, dynamic constitutive models are usually used to describe its ratedependent properties. Here, the dynamic increase factor (DIF) model is used to analyze the interfacial bonding performance of the FRP-concrete considering the strain rate effect. There are several types of DIF model widely used, such as the power function model proposed by CEB code [30] and the logarithmic function model proposed by Kulkarni and Shah (K \& S model) [24]. The latter one can be expressed as follows:

$$
T_{\mathrm{DIF}}=\frac{\sigma_{d}}{\sigma_{s}}=\alpha \ln \left(\frac{\dot{\varepsilon}_{d}}{\dot{\varepsilon}_{s}}\right)+\beta,
$$

where $\dot{\varepsilon}_{d}$ and $\dot{\varepsilon}_{s}$ are dynamic strain rate and quasistatic strain rate, respectively. $\sigma_{d}$ and $\sigma_{s}$ are the dynamic and quasistatic compressive strength of the concrete, respectively. $\alpha$ and $\beta$ are the fitting parameters.

Here, the $\mathrm{K} \& \mathrm{~S}$ model is used to conduct the nonlinear regression fitting analysis on $G_{f}$ and $\tau_{\max }$ to investigate the strain rate effect on the interfacial bearing capacity between the FRP and the concrete. For simplicity, only the effect of concrete strength is considered in this model. For the data comparison, the normalized interfacial energy at break $\left(G_{f d} / G_{f s}\right)$ and peak shear stress $\left(\tau_{m d} / \tau_{m s}\right)$ are used as the ordinate and the relative stain rate $\left(\dot{\varepsilon}_{d} / \dot{\varepsilon}_{s}\right)$ as the abscissa. The specific model is expressed as follows:

$$
\begin{aligned}
& \frac{G_{f d}}{G_{f s}}=\alpha \ln \left(\frac{\dot{\varepsilon}_{d}}{\dot{\varepsilon}_{s}}\right)+\beta, \\
& \frac{\tau_{m d}}{\tau_{m s}}=\gamma \ln \left(\frac{\dot{\varepsilon}_{d}}{\dot{\varepsilon}_{s}}\right)+\omega,
\end{aligned}
$$

where $G_{f d}$ and $G_{f s}$ are the interfacial fracture energy under the dynamic and the quasistatic load, respectively. $\tau_{m s}$ and $\tau_{m s}$ are the corresponding peak shear stresses. $\alpha, \beta, \gamma$, and $\omega$ are the parameters obtained by the nonlinear regression of the experimental results.

The fitting curves of the interface strain rate effect model for the specimens using C20A, C30A, and C40A are shown in Figure 10 with the fitting parameters and the value of $R^{2}$. It is observed that the correlation coefficients are over 0.9 for all 


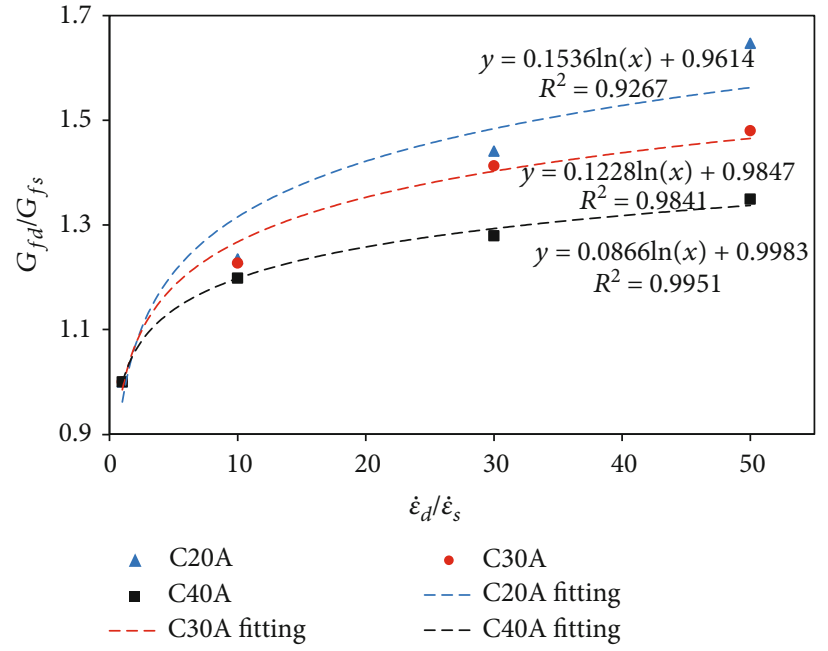

(a) $G_{f}$

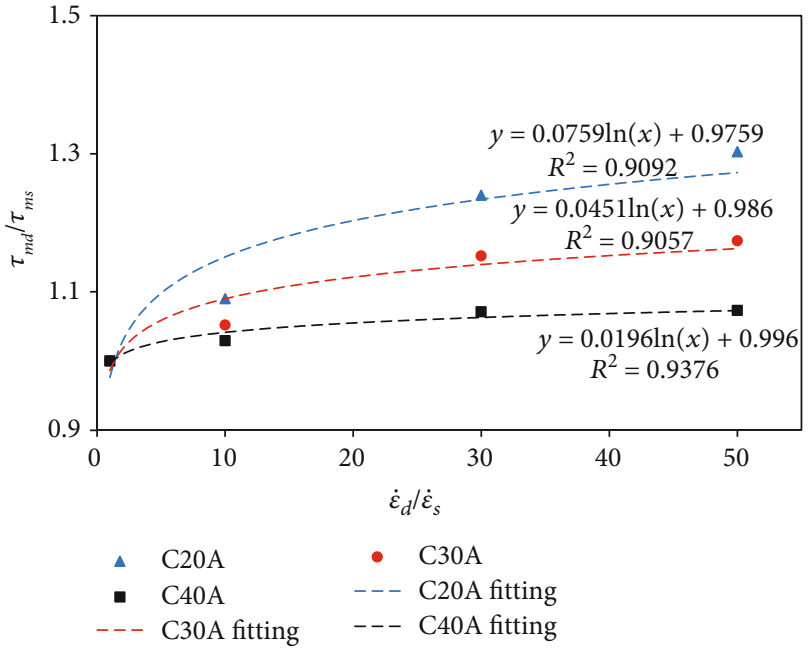

(b) $\tau_{\max }$

FIGURE 10: Normalized interfacial strain rate effect model.

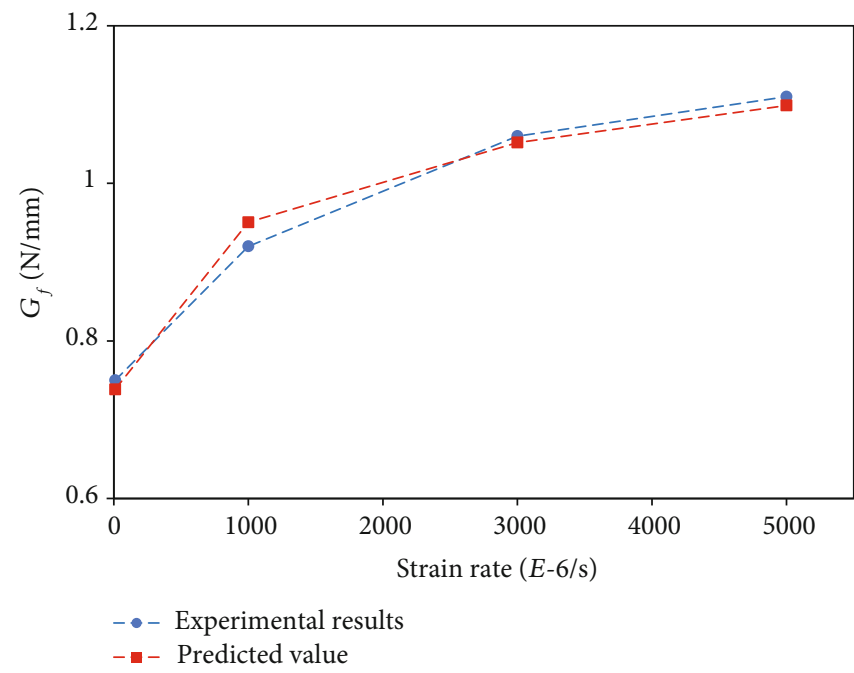

(a) $G_{f}$

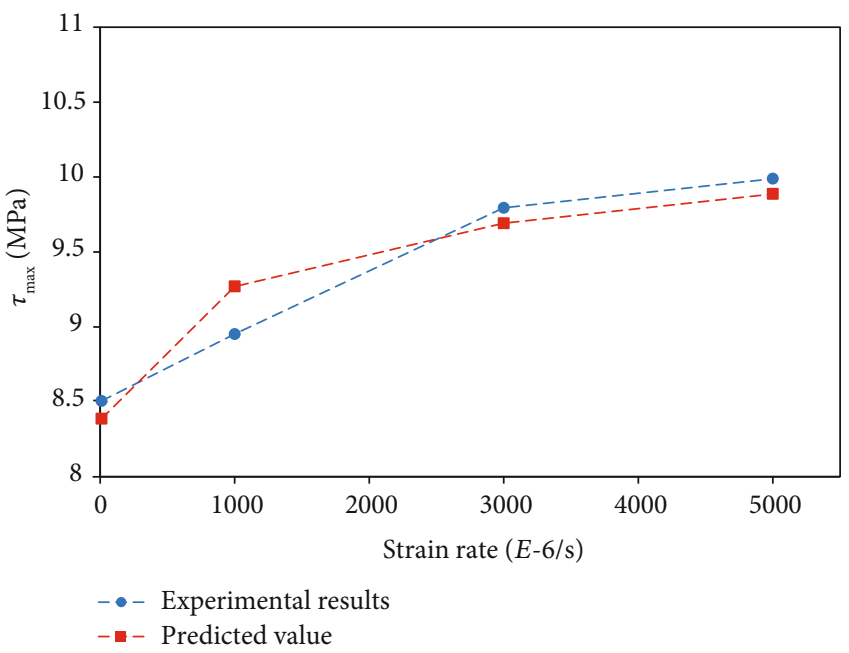

(b) $\tau_{\max }$

FIgURE 11: Comparison of $G_{f}$ and $\tau_{\max }$ between experimental and estimated values.

fitting curves, indicating that the proposed model performs well to predict the strain rate effect on the interfacial fracture energy and the peak shear stress.

The normalized strain rate effect model shown in Figure 10 can be regarded as the dynamic increase factor derived from the ratio of the dynamic constitutive model to the static constitutive model. In order to obtain a reasonable estimation of $G_{f}$ and $\tau_{\max }$, Equations (8) and (9) are rewritten as follows:

$$
\begin{aligned}
G_{f d} & =G_{f s}\left(\alpha \ln \left(\frac{\dot{\varepsilon}_{d}}{\dot{\varepsilon}_{s}}\right)+\beta\right), \\
\tau_{m d} & =\tau_{m s}\left(\gamma \ln \left(\frac{\dot{\varepsilon}_{d}}{\dot{\varepsilon}_{s}}\right)+\omega\right) .
\end{aligned}
$$

To verify the accuracy of the estimating model, $G_{f}$ and $\tau_{\max }$ for the specimens of $\mathrm{C} 30 \mathrm{~A}$ obtained by the estimating model and the experiments are compared in Figure 11. For $G_{f}$, the relative errors of estimation under strain rates I, II, III, and IV are $1.5 \%, 3.3 \%, 0.7 \%$, and $1.0 \%$, respectively. For $\tau_{\text {max }}$, the relative errors become $1.4 \%, 3.5 \%, 1.1 \%$, and $1.0 \%$, respectively. Consequently, the estimated values agree well with the experimental observations.

\section{Conclusion}

The interfacial debonding mechanism between FRP sheet and concrete substrate under medium strain rate (from 1.0 $E-4 / \mathrm{s}$ to $5.0 E-3 / \mathrm{s}$ ) is thoroughly investigated by the single-lap shear tests. The influences of the strain rate, 
concrete strength, FRP, and adhesive type on the interfacial shear capacity are analyzed by linear and nonlinear fitting of experimental results. It is found that the interfacial fracture energy and the peak shear stress significantly increase with the strain rate and concrete strength. Owing to the ductility of BFRP, the interfacial fracture energy of the specimens using BFRP is higher than those using CFRP, but their trends with the increasing strain rate are similar. The adhesive with lower elastic modulus is helpful to improve interfacial energy dissipation under dynamic load. Within the range of the strain rate concerned in this paper, the effective bond length is between $75 \mathrm{~mm}$ and $90 \mathrm{~mm}$, which deceases with the concrete strength and the strain rate. A logarithmic function model, in the form of $\mathrm{K} \& \mathrm{~S}$ model, is proposed to estimate the interfacial fracture energy and the peak shear stress under medium strain rate, and good agreements are observed between the estimated values and experimental results. It should be pointed out that the proposed fracture energybased model is able to indirectly evaluate the macroscopic mechanical properties of the interface, but the local situation of the interface cannot be investigated in detail due to the lack of stress/strain field description. Consequently, more advanced techniques, such as digital image correlation (DIC) technique, are required for further study.

\section{Data Availability}

The experimental data of interfacial debonding between FRP and concrete substrate used to support the findings of this study are available from the corresponding author upon request.

\section{Conflicts of Interest}

The authors declare that they do not have any commercial or associative interest that represents a conflict of interest in connection with the work submitted.

\section{Acknowledgments}

The authors would like to appreciate the financial support of the National Natural Science Foundation of China (Grant No. 51308307), the Key Laboratory of Impact and Safety Engineering (Ningbo University), Ministry of Education (Grant No. CJ201914), the Zhejiang Provincial Natural Science Foundation of China (Grant No. LY18E080013), and K.C. Wong Magna Fund in Ningbo University.

\section{References}

[1] R. A. Barnes and G. C. Mays, "Strengthening of reinforced concrete beams in shear by the use of externally bonded steel plates: part 2 - design guidelines," Construction and Building Materials, vol. 20, no. 6, pp. 403-411, 2006.

[2] S. Park, T. Kim, K. Kim, and S. N. Hong, "Flexural behavior of steel I-beam prestressed with externally unbonded tendons," Journal of Constructional Steel Research, vol. 66, no. 1, pp. 125-132, 2010.

[3] M. Elghazy, A. el Refai, U. Ebead, and A. Nanni, "Experimental results and modelling of corrosion-damaged concrete beams strengthened with externally-bonded composites," Engineering Structures, vol. 172, pp. 172-186, 2018.

[4] X. Zhang, Z. Wu, and Y. Cheng, “An approach of steel plate hybrid bonding technique to externally bonded fibrereinforced polymer strengthening system," International Journal of Distributed Sensor Networks, vol. 14, no. 6, 2018.

[5] J. F. Chen and J. G. Teng, "Anchorage strength models for FRP and steel plates bonded to concrete," Journal of Structural Engineering, vol. 127, no. 7, pp. 784-791, 2001.

[6] S. K. Woo and Y. Lee, "Experimental study on interfacial behavior of CFRP-bonded concrete," KSCE Journal of Civil Engineering, vol. 14, no. 3, pp. 385-393, 2010.

[7] W. Elsayed, U. A. Ebead, and K. W. Neale, "Interfacial behavior and debonding failures in FRP-strengthened concrete slabs," Journal of Composites for Construction, vol. 11, no. 6, pp. 619-628, 2007.

[8] P. Cornetti and A. Carpinteri, "Modelling the FRP-concrete delamination by means of an exponential softening law," Engineering Structures, vol. 33, no. 6, pp. 1988-2001, 2011.

[9] H. Toutanji, P. Saxena, L. Zhao, and T. Ooi, "Prediction of interfacial bond failure of FRP-concrete surface," Journal of Composites for Construction, vol. 11, no. 4, pp. 427-436, 2007.

[10] M. L. Bennegadi, K. Hadjazi, Z. Sereir, S. Amziane, and B. el Mahi, "General cohesive zone model for prediction of interfacial stresses induced by intermediate flexural crack of FRP-plated RC beams," Engineering Structures, vol. 126, pp. 147-157, 2016.

[11] X. Z. Lu, L. P. Ye, J. G. Teng, and J. J. Jiang, "Meso-scale finite element model for FRP sheets/plates bonded to concrete," Engineering Structures, vol. 27, no. 4, pp. 564-575, 2005.

[12] S. T. Smith and J. G. Teng, "FRP-strengthened RC beams. I: review of debonding strength models," Engineering Structures, vol. 24, no. 4, pp. 385-395, 2002.

[13] R. Kalfat, R. Al-Mahaidi, and S. T. Smith, "Anchorage devices used to improve the performance of reinforced concrete beams retrofitted with FRP composites: state-of-the-art review," Journal of Composites for Construction, vol. 17, no. 1, pp. 14-33, 2013.

[14] S. A. Hadigheh, S. S. Mahini, and M. R. Maheri, "Seismic behavior of FRP-retrofitted reinforced concrete frames," Journal of Earthquake Engineering, vol. 18, no. 8, pp. 1171-1197, 2014.

[15] A. A. Mutalib and H. Hao, "Development of P-I diagrams for FRP strengthened RC columns," International Journal of Impact Engineering, vol. 38, no. 5, pp. 290-304, 2011.

[16] M. Saatcioglu, T. Ozbakkaloglu, N. Naumoski, and A. Lloyd, "Response of earthquake-resistant reinforced-concrete buildings to blast loading," Canadian Journal of Civil Engineering, vol. 36, no. 8, pp. 1378-1390, 2009.

[17] P. H. Bischoff and S. H. Perry, "Compressive behaviour of concrete at high strain rates," Materials and Structures, vol. 24, no. 6, pp. 425-450, 1991.

[18] J. W. Shi, H. Zhu, Z. S. Wu, and G. Wu, "Experimental study of the strain rate of FRP sheet-concrete interface," China Civil Engineering Journal, vol. 45, no. 12, pp. 99-107, 2012.

[19] D. Shen, H. Shi, Y. Ji, and F. Yin, "Strain rate effect on effective bond length of basalt FRP sheet bonded to concrete," Construction and Building Materials, vol. 82, pp. 206-218, 2015. 
[20] D. Shen, X. Shi, Y. Ji, and F. Yin, "Strain rate effect on bond stress-slip relationship between basalt fiber-reinforced polymer sheet and concrete," Journal of Reinforced Plastics and Composites, vol. 34, no. 7, pp. 547-563, 2015.

[21] A. Caggiano, E. Martinelli, D. Said Schicchi, and G. Etse, "A modified Duvaut-Lions zero-thickness interface model for simulating the rate-dependent bond behavior of FRPconcrete joints," Composites Part B: Engineering, vol. 149, pp. 260-267, 2018.

[22] G. M. Chen, J. G. Teng, J. F. Chen, and Q. G. Xiao, "Finite element modeling of debonding failures in FRP-strengthened RC beams: a dynamic approach," Computers and Structures, vol. 158, pp. 167-183, 2015.

[23] X. Lin and Y. X. Zhang, "Nonlinear finite element analysis of FRP-strengthened reinforced concrete panels under blast loads," International Journal of Computational Methods, vol. 13, no. 4, article 1641002, 2016.

[24] S. M. Kulkarni and S. P. Shah, "Response of reinforced concrete beams at high strain rates," ACI Structural Journal, vol. 95, no. 6, pp. 705-715, 1998.

[25] B. Wan, C. Jiang, and Y. F. Wu, "Effect of defects in externally bonded FRP reinforced concrete," Construction and Building Materials, vol. 172, pp. 63-76, 2018.

[26] J. G. Dai, T. Ueda, and Y. Sato, "Unified analytical approaches for determining shear bond characteristics of FRP-concrete interfaces through pullout tests," Journal of Advanced Concrete Technology, vol. 4, no. 1, pp. 133-145, 2006.

[27] J. G. Dai, T. Ueda, and Y. Sato, "Development of the nonlinear bond stress-slip model of fiber reinforced plastics sheetconcrete interfaces with a simple method," Journal of Composites for Construction, vol. 9, no. 1, pp. 52-62, 2005.

[28] M. J. Chajes, W. W. Finch Jr., T. F. Januszka, and T. A. Thomson Jr., "Bond and force transfer of compositematerial plates bonded to concrete," ACI Structural Journal, vol. 93, no. 2, pp. 208-217, 1996.

[29] T. C. Triantafillou, "Fracture mechanics approaches to concrete strengthening using FRP materials," in Facture Mechanics of Concrete Structures Proceedings FRAMCOS-3, pp. 1761-1770, 1998.

[30] Comité Euro-International du Béton, CEB-FIP model code 1990, Redwood Books, Trowbridge, Wiltshire, UK, 1993. 


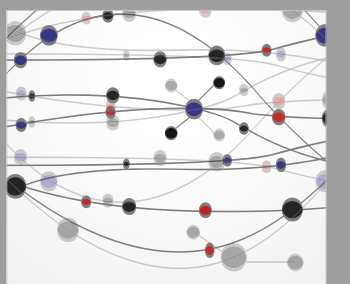

The Scientific World Journal
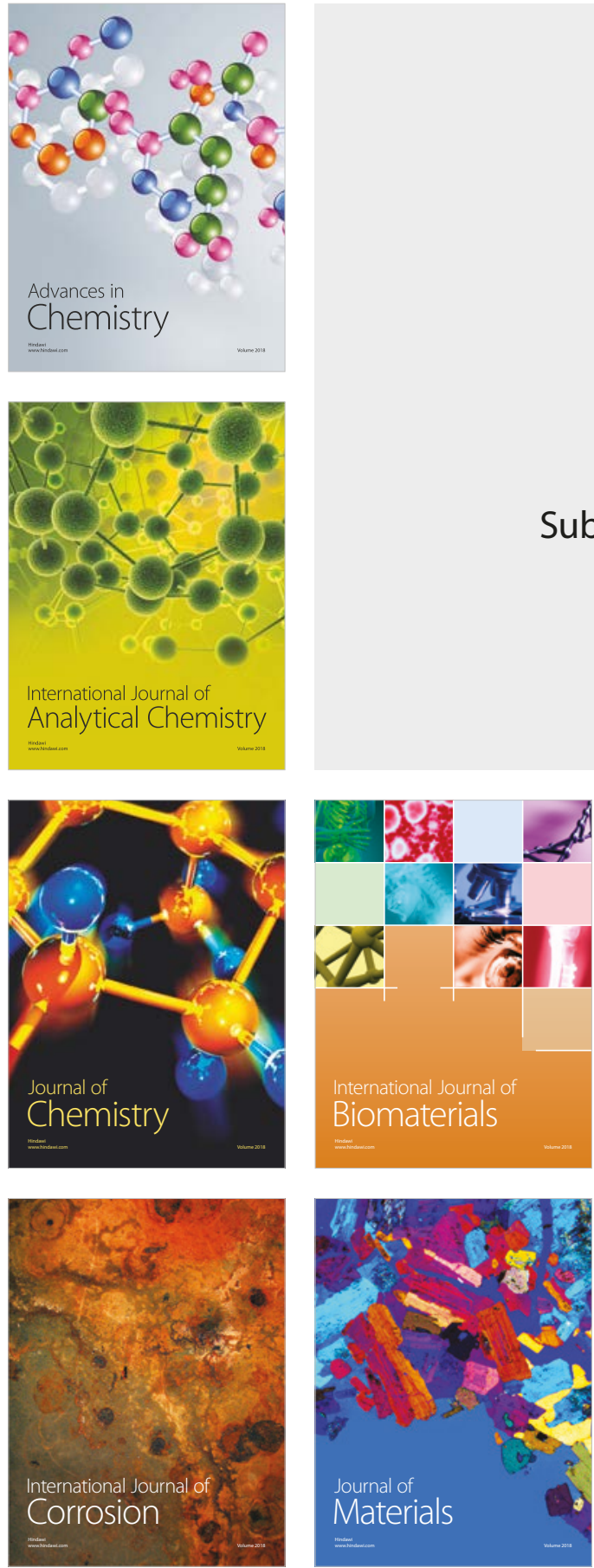

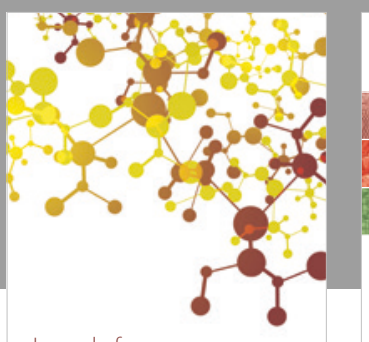

Journal of

Applied Chemistry
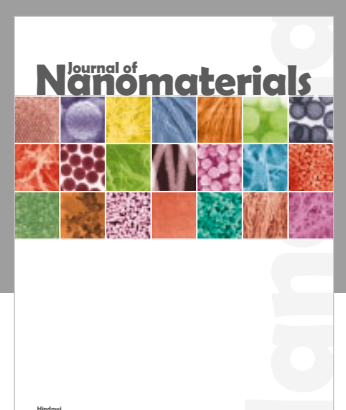

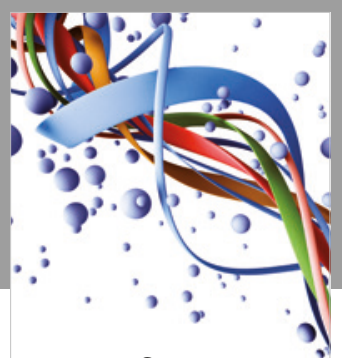

Scientifica

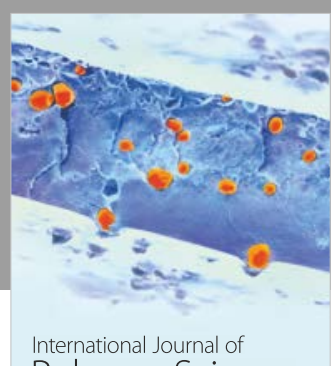

Polymer Science

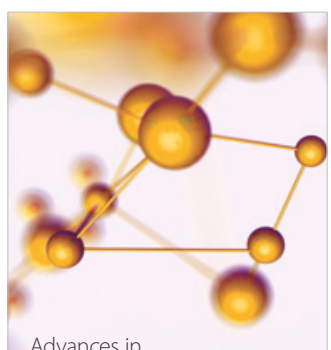

Physical Chemistry
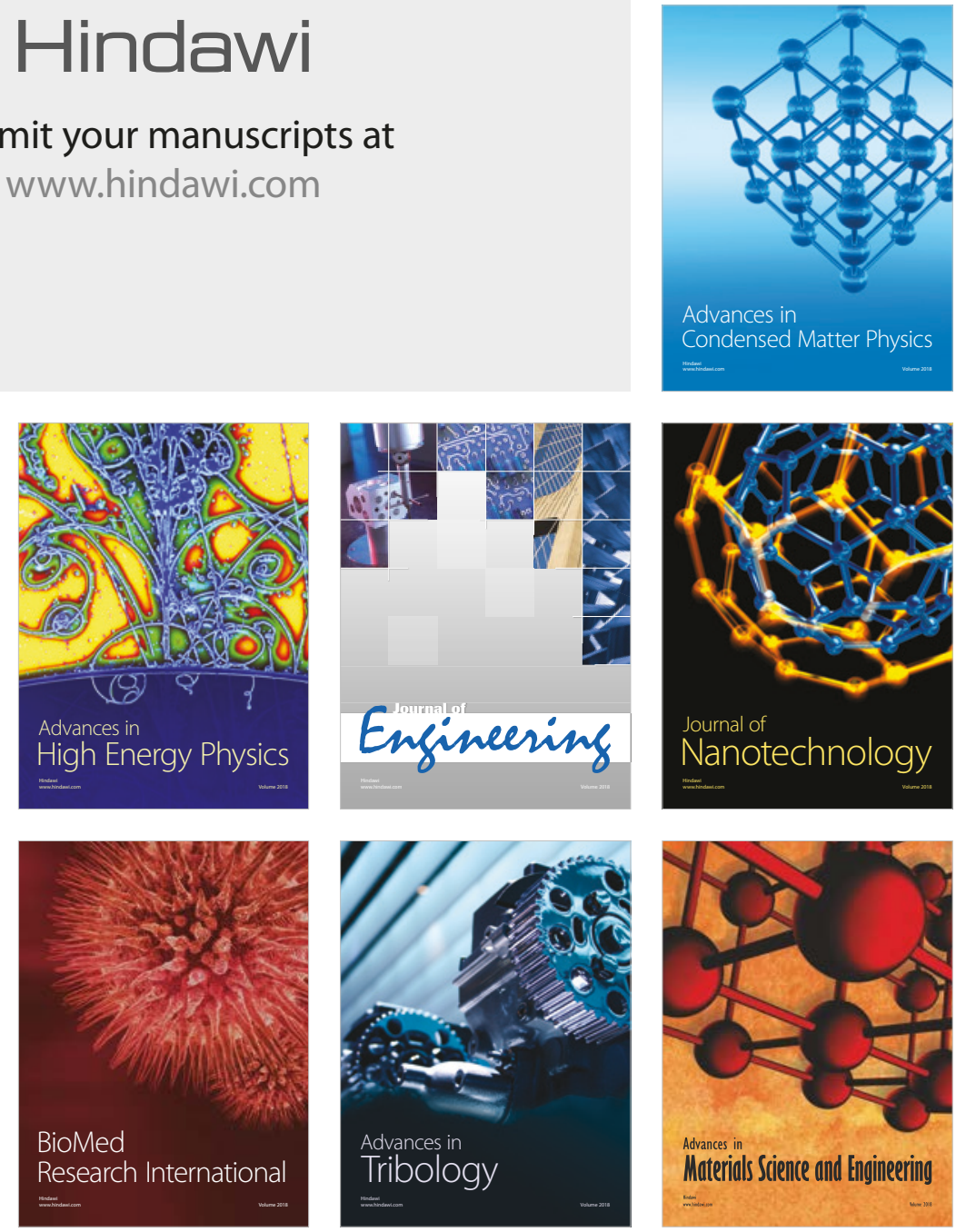